\title{
0543 HATE VIOLENCE: EMERGENCY DEPARTMENT PERSPECTIVE
}

V Sivarajasingam*, S Read, M Svobodova, L Wight, J Shepherd Correspondence: Cardiff University, Violence and Society Research Group School of Dentistry Heath Park Cardiff CF14 4XY, UK

\subsection{6/ip.2010.029215.543}

Purpose The purpose of this study was to investigate the status and potential of Emergency Department (ED) data for identifying and preventing hate violence.

Methods Face-to-face interviews with patients attending three EDs in England and Wales (Blackburn, Cardiff and Leicester) with injuries sustained in violence were undertaken; 120 semistructured interviews, 40 in each centre. Interviews were also conducted with Crime and Disorder Reduction Partnership/ Community Safety Partnership members and ED staff to evaluate violence data sharing protocols. All interviews were audio 


\section{IP Safety 2010 abstracts}

recorded, transcribed and uploaded onto OSR Internationals NVivo 8 software for analysis.

Results The 124 respondents comprised 99 men (79.8\%) and 25 women $(20.2 \%) ; 55$ were aged $16-24$ years (44.4\%). The ethnic group most often represented were White British, English, Irish or Scottish (103 respondents; 83.0\%). Altogether, there were eight definite cases of hate violence, accounting for $6.5 \%$ of all assaults. Seven of these were racially and religiously aggravated (5.6\% of all assaults), while one ( $0.9 \%$ ) was aggravated by sexual orientation. Alcohol consumption was a contributing factor in $75 \%$ of hate violence. In two of the three centres studied, ED violence data were not being utilised for violence prevention.

Conclusions From these findings, alcohol intoxication promotes hate violence. Although EDs are a valuable source of information about this problem routine collection of the relevant data is not practical, reflecting the need for substantial time and qualitative interview skills which are not available in EDs. 